\title{
A Simple Proof of Sklyanin's Formula for Canonical Spectral Coordinates of the Rational Calogero-Moser System
}

Tamás F. GÖRBE

Department of Theoretical Physics, University of Szeged, Tisza Lajos krt 84-86, H-6720 Szeged, Hungary

E-mail:tfgorbe@physx.u-szeged.hu

URL: http://www.staff.u-szeged.hu/ tfgorbe/

Received January 19, 2016, in final form March 08, 2016; Published online March 11, 2016

http://dx.doi.org/10.3842/SIGMA.2016.027

\begin{abstract}
We use Hamiltonian reduction to simplify Falqui and Mencattini's recent proof of Sklyanin's expression providing spectral Darboux coordinates of the rational CalogeroMoser system. This viewpoint enables us to verify a conjecture of Falqui and Mencattini, and to obtain Sklyanin's formula as a corollary.
\end{abstract}

Key words: integrable systems; Calogero-Moser type systems; spectral coordinates; Hamiltonian reduction; action-angle duality

2010 Mathematics Subject Classification: 14H70; 37J15; 53D20

\section{Introduction}

Integrable many-body systems in one spatial dimension form an important class of exactly solvable Hamiltonian systems with their diverse mathematical structure and widespread applicability in physics $[2,6,10]$. Among these many-body systems, one of the most widely known is the rational Calogero-Moser model of equally massive interacting particles moving along a line with a pair potential inversely proportional to the square of the distance. The model was introduced and solved at the quantum level by Calogero [1]. The complete integrability of its classical version was established by Moser [5], who employed the Lax formalism to identify a complete set of commuting integrals as coefficients of the characteristic polynomial of a certain Hermitian matrix function, called the Lax matrix.

These developments might prompt one to consider the Poisson commuting eigenvalues of the Lax matrix and be interested in searching for an expression of conjugate variables. Such an expression was indeed formulated by Sklyanin [8] in his work on bispectrality, and worked out in detail for the open Toda chain [9]. Sklyanin's formula for the rational Calogero-Moser model was recently confirmed within the framework of bi-Hamiltonian geometry by Falqui and Mencattini [3] in a somewhat circuitous way, although a short-cut was pointed out in the form of a conjecture. The purpose of this paper is to prove this conjecture and offer an alternative simple proof of Sklyanin's formula using results of Hamiltonian reduction.

Section 2 is a recap of complete integrability and action-angle duality for the rational Calogero-Moser system in the context of Hamiltonian reduction. In Section 3 we put these ideas into practice when we identify the canonical variables of [3] in terms of the reduction picture, and prove the relation conjectured in that paper. We attain Sklyanin's formula as a corollary. Section 4 contains our concluding remarks on possible generalizations. 


\section{The rational Calogero-Moser system via reduction}

We begin by describing the rational Calogero-Moser system and recalling how it originates from Hamiltonian reduction [4]. The content of this section is standard and only included for the sake of self-consistency.

For $n$ particles, let the $n$-tuples $q=\left(q_{1}, \ldots, q_{n}\right)$ and $p=\left(p_{1}, \ldots, p_{n}\right)$ collect their coordinates and momenta, respectively. Then the Hamiltonian of the model reads

$$
H(q, p)=\frac{1}{2} \sum_{j=1}^{n} p_{j}^{2}+g^{2} \sum_{\substack{j, k=1 \\ j<k)}}^{n} \frac{1}{\left(q_{j}-q_{k}\right)^{2}},
$$

where $g$ is a real coupling constant tuning the strength of particle interaction. The pair potential is singular at $q_{j}=q_{k}(j \neq k)$, hence any initial ordering of the particles remains unchanged during time-evolution. The configuration space is chosen to be the domain $\mathcal{C}=\left\{q \in \mathbb{R}^{n} \mid q_{1}>\cdots>q_{n}\right\}$, and the phase space is its cotangent bundle

$$
T^{*} \mathcal{C}=\left\{(q, p) \mid q \in \mathcal{C}, p \in \mathbb{R}^{n}\right\},
$$

endowed with the standard symplectic form

$$
\omega=\sum_{j=1}^{n} d q_{j} \wedge d p_{j}
$$

The Hamiltonian system $\left(T^{*} \mathcal{C}, \omega, H\right)$, called the rational Calogero-Moser system, can be obtained as an appropriate Marsden-Weinstein reduction of the free particle moving in the space of $n \times n$ Hermitian matrices as follows.

Consider the manifold of pairs of $n \times n$ Hermitian matrices

$$
M=\left\{(X, P) \mid X, P \in \mathfrak{g l}(n, \mathbb{C}), X^{\dagger}=X, P^{\dagger}=P\right\},
$$

equipped with the symplectic form

$$
\Omega=\operatorname{tr}(d X \wedge d P) .
$$

The Hamiltonian of the analogue of a free particle reads

$$
\mathcal{H}(X, P)=\frac{1}{2} \operatorname{tr}\left(P^{2}\right) .
$$

The equations of motion can be solved explicitly for this Hamiltonian system $(M, \Omega, \mathcal{H})$, and the general solution is given by $X(t)=t P_{0}+X_{0}, P(t)=P_{0}$. Moreover, the functions $\mathcal{H}_{k}(X, P)=$ $\frac{1}{k} \operatorname{tr}\left(P^{k}\right), k=1, \ldots, n$ form an independent set of commuting first integrals.

The group of $n \times n$ unitary matrices $U(n)$ acts on $M(4)$ by conjugation

$$
(X, P) \rightarrow\left(U X U^{\dagger}, U P U^{\dagger}\right), \quad U \in U(n),
$$

leaves both the symplectic form $\Omega(5)$ and the Hamiltonians $\mathcal{H}_{k}$ invariant, and the matrix commutator $(X, P) \rightarrow[X, P]$ is a momentum map for this $U(n)$-action. Consider the Hamiltonian reduction performed by factorizing the momentum constraint surface

$$
[X, P]=\mathrm{i} g\left(v v^{\dagger}-\mathbf{1}_{n}\right)=: \mu, \quad v=(1 \ldots 1)^{\dagger} \in \mathbb{R}^{n}, \quad g \in \mathbb{R},
$$


with the stabilizer subgroup $G_{\mu} \subset U(n)$ of $\mu$, e.g., by diagonalization of the $X$ component. This yields the gauge slice $S=\left\{(Q(q, p), L(q, p)) \mid q \in \mathcal{C}, p \in \mathbb{R}^{n}\right\}$, where

$$
\begin{aligned}
Q_{j k} & =\left(U X U^{\dagger}\right)_{j k}=q_{j} \delta_{j k}, \\
L_{j k} & =\left(U P U^{\dagger}\right)_{j k}=p_{j} \delta_{j k}+\mathrm{i} g \frac{1-\delta_{j k}}{q_{j}-q_{k}}, \quad j, k=1, \ldots, n .
\end{aligned}
$$

This $S$ is symplectomorphic to the reduced phase space and to $T^{*} \mathcal{C}(2)$ since it inherits the reduced symplectic form $\omega(3)$. The unreduced Hamiltonians project to a commuting set of independent integrals $H_{k}=\frac{1}{k} \operatorname{tr}\left(L^{k}\right), k=1, \ldots, n$, such that $H_{2}=H$ (1) and what's more, the completeness of Hamiltonian flows follows automatically from the reduction. Therefore the rational Calogero-Moser system is completely integrable.

The similar role of matrices $X$ and $P$ in the derivation above can be exploited to construct action-angle variables for the rational Calogero-Moser system. This is done by switching to the gauge, where the $P$ component is diagonalized by some matrix $\tilde{U} \in G_{\mu}$, and it boils down to the gauge slice $\tilde{S}=\left\{(\tilde{Q}(\phi, \lambda), \tilde{L}(\phi, \lambda)) \mid \phi \in \mathbb{R}^{n}, \lambda \in \mathcal{C}\right\}$, where

$$
\begin{aligned}
& \tilde{Q}_{j k}=\left(\tilde{U} X \tilde{U}^{\dagger}\right)_{j k}=\phi_{j} \delta_{j k}-\mathrm{i} g \frac{1-\delta_{j k}}{\lambda_{j}-\lambda_{k}}, \\
& \tilde{L}_{j k}=\left(\tilde{U} P \tilde{U}^{\dagger}\right)_{j k}=\lambda_{j} \delta_{j k}, \quad j, k=1, \ldots, n .
\end{aligned}
$$

By construction, $\tilde{S}$ with the symplectic form $\tilde{\omega}=\sum_{j=1}^{n} d \phi_{j} \wedge d \lambda_{j}$ is also symplectomorphic to the reduced phase space, thus a canonical transformation $(q, p) \rightarrow(\phi, \lambda)$ is obtained, where the reduced Hamiltonians depend only on $\lambda$, viz. $H_{k}=\frac{1}{k}\left(\lambda_{1}^{k}+\cdots+\lambda_{n}^{k}\right), k=1, \ldots, n$.

\section{Sklyanin's formula}

Now, we turn to the question of variables conjugate to the Poisson commuting eigenvalues $\lambda_{1}, \ldots, \lambda_{n}$ of $L(6)$, i.e., such functions $\theta_{1}, \ldots, \theta_{n}$ in involution that

$$
\left\{\theta_{j}, \lambda_{k}\right\}=\delta_{j k}, \quad j, k=1, \ldots, n .
$$

At the end of Section 2 we saw that the variables $\phi_{1}, \ldots, \phi_{n}$ are such functions. These actionangle variables $\lambda, \phi$ were already obtained by Moser [5] using scattering theory, and also appear in Ruijsenaars' proof of the self-duality of the rational Calogero-Moser system [7].

Let us define the following functions over the phase space $T^{*} \mathcal{C}(2)$ with dependence on an additional variable $z$ :

$$
\begin{aligned}
& A(z)=\operatorname{det}\left(z \mathbf{1}_{n}-L\right), \quad C(z)=\operatorname{tr}\left(Q \operatorname{adj}\left(z \mathbf{1}_{n}-L\right) v v^{\dagger}\right), \\
& D(z)=\operatorname{tr}\left(Q \operatorname{adj}\left(z \mathbf{1}_{n}-L\right)\right),
\end{aligned}
$$

where $Q$ and $L$ are given by (6), $v=(1 \ldots 1)^{\dagger} \in \mathbb{R}^{n}$ and adj denotes the adjugate matrix, i.e., the transpose of the cofactor matrix. Sklyanin's formula [8] for $\theta_{1}, \ldots, \theta_{n}$ then reads

$$
\theta_{k}=\frac{C\left(\lambda_{k}\right)}{A^{\prime}\left(\lambda_{k}\right)}, \quad k=1, \ldots, n .
$$

In [3] Falqui and Mencattini have shown that

$$
\mu_{k}=\frac{D\left(\lambda_{k}\right)}{A^{\prime}\left(\lambda_{k}\right)}, \quad k=1, \ldots, n
$$


are conjugate variables to $\lambda_{1}, \ldots, \lambda_{n}$, and

$$
\theta_{k}=\mu_{k}+f_{k}\left(\lambda_{1}, \ldots, \lambda_{n}\right), \quad k=1, \ldots, n,
$$

with such $\lambda$-dependent functions $f_{1}, \ldots, f_{n}$ that

$$
\frac{\partial f_{j}}{\partial \lambda_{k}}=\frac{\partial f_{k}}{\partial \lambda_{j}}, \quad j, k=1, \ldots, n
$$

thus $\theta_{1}, \ldots, \theta_{n}$ given by Sklyanin's formula (9) are conjugate to $\lambda_{1}, \ldots, \lambda_{n}$. This was done in a roundabout way, although the explicit form of relation (11) was conjectured.

Here we take a different route by making use of the reduction viewpoint of Section 2. From this perspective, the problem becomes transparent and can be solved effortlessly. First, we show that $\mu_{1}, \ldots, \mu_{n}(10)$ are nothing else than the angle variables $\phi_{1}, \ldots, \phi_{n}$.

Lemma. The variables $\mu_{1}, \ldots, \mu_{n}$ defined in (10) are the angle variables $\phi_{1}, \ldots, \phi_{n}$ of the rational Calogero-Moser system.

Proof. Notice that, by definition, $\mu_{1}, \ldots, \mu_{n}$ are gauge invariant, thus by working in the gauge, where the $P$ component is diagonal, that is with the matrices $\tilde{Q}, \tilde{L}(7)$, we get

$$
\frac{D(z)}{A^{\prime}(z)}=\frac{\sum_{j=1}^{n} \phi_{j} \prod_{\substack{\ell=1 \\ \ell \neq j}}^{n}\left(z-\lambda_{\ell}\right)}{\sum_{j=1}^{n} \prod_{\substack{\ell=1 \\ \ell \neq j}}^{n}\left(z-\lambda_{\ell}\right)} .
$$

Substituting $z=\lambda_{k}$ into (13) yields $\mu_{k}=\phi_{k}$, for each $k=1, \ldots, n$.

Next, we prove the relation of functions $A, C, D(8)$, that was conjectured in [3].

Theorem. For any $n \in \mathbb{N},(q, p) \in T^{*} \mathcal{C}(2)$, and $z \in \mathbb{C}$ we have

$$
C(z)=D(z)+\frac{\mathrm{i} g}{2} A^{\prime \prime}(z)
$$

Proof. Pick any point $(q, p)$ in the phase space $T^{*} \mathcal{C}$ and consider the corresponding point $(\lambda, \phi)$ in the space of action-angle variables. Since $A(z)=\left(z-\lambda_{1}\right) \cdots\left(z-\lambda_{n}\right)$ we have

$$
\frac{\mathrm{i} g}{2} A^{\prime \prime}(z)=\mathrm{i} g \sum_{\substack{j, k=1 \\ j<k)}}^{n} \prod_{\substack{\ell=1 \\ \ell \neq j, k)}}^{n}\left(z-\lambda_{\ell}\right) .
$$

The difference of functions $C$ and $D(8)$ reads

$$
C(z)-D(z)=\operatorname{tr}\left(Q \operatorname{adj}\left(z \mathbf{1}_{n}-L\right)\left(v v^{\dagger}-\mathbf{1}_{n}\right)\right) .
$$

Due to gauge invariance, we are allowed to work with $\tilde{Q}, \tilde{L}(7)$ instead of $Q, L(6)$. Therefore (14) can be written as the sum of all off-diagonal components of $\tilde{Q} \operatorname{adj}\left(z \mathbf{1}_{n}-\tilde{L}\right)$, that is

$$
C(z)-D(z)=\mathrm{i} g \sum_{\substack{j, k=1 \\ j \neq k)}}^{n} \frac{-1}{\lambda_{j}-\lambda_{k}} \prod_{\substack{\ell=1 \\ \ell \neq k)}}^{n}\left(z-\lambda_{\ell}\right)=\mathrm{i} g \sum_{\substack{j, k=1 \\ j<k)}}^{n} \prod_{\substack{\ell=1 \\ \ell \neq j, k)}}^{n}\left(z-\lambda_{\ell}\right) .
$$

This concludes the proof. 
Our theorem confirms that indeed relation (11) is valid with

$$
f_{k}\left(\lambda_{1}, \ldots, \lambda_{n}\right)=\frac{\mathrm{i} g}{2} \frac{A^{\prime \prime}\left(\lambda_{k}\right)}{A^{\prime}\left(\lambda_{k}\right)}=\mathrm{i} g \sum_{\substack{\ell=1 \\ \ell \neq k)}}^{n} \frac{1}{\lambda_{k}-\lambda_{\ell}}, \quad k=1, \ldots, n,
$$

for which (12) clearly holds. An immediate consequence, as we indicated before, is that $\theta_{1}, \ldots, \theta_{n}$ (9) are conjugate variables to $\lambda_{1}, \ldots, \lambda_{n}$, thus Sklyanin's formula is verified.

Corollary (Sklyanin's formula). The variables $\theta_{1}, \ldots, \theta_{n}$ defined by

$$
\theta_{k}=\frac{C\left(\lambda_{k}\right)}{A^{\prime}\left(\lambda_{k}\right)}, \quad k=1, \ldots, n
$$

are conjugate to the eigenvalues $\lambda_{1}, \ldots, \lambda_{n}$ of the Lax matrix $L$.

\section{Discussion}

There seem to be several ways for generalization. For example, one might consider rational Calogero-Moser models associated to root systems other than type $A_{n-1}$. The hyperbolic Calogero-Moser systems as well as, the 'relativistic' Calogero-Moser systems, also known as Ruijsenaars-Schneider systems, are also of considerable interest.

\section{Acknowledgements}

Thanks are due to László Fehér for drawing our attention to the duality perspective. This work was supported in part by the Hungarian Scientific Research Fund (OTKA) under the grant K-111697. The work was also partially supported by COST (European Cooperation in Science and Technology) in COST Action MP1405 QSPACE.

\section{References}

[1] Calogero F., Solution of the one-dimensional $N$-body problems with quadratic and/or inversely quadratic pair potentials, J. Math. Phys. 12 (1971), 419-436, Erratum, J. Math. Phys. 37 (1996), 3646.

[2] Calogero F., Classical many-body problems amenable to exact treatments, Lecture Notes in Physics. New Series m: Monographs, Vol. 66, Springer-Verlag, Berlin, 2001.

[3] Falqui G., Mencattini I., Bi-Hamiltonian geometry and canonical spectral coordinates for the rational Calogero-Moser system, arXiv:1511.06339.

[4] Kazhdan D., Kostant B., Sternberg S., Hamiltonian group actions and dynamical systems of Calogero type, Comm. Pure Appl. Math. 31 (1978), 481-507.

[5] Moser J., Three integrable Hamiltonian systems connected with isospectral deformations, Adv. Math. 16 (1975), 197-220.

[6] Perelomov A.M., Integrable systems of classical mechanics and Lie algebras. Vol. I, Birkhäuser Verlag, Basel, 1990.

[7] Ruijsenaars S.N.M., Action-angle maps and scattering theory for some finite-dimensional integrable systems. I. The pure soliton case, Comm. Math. Phys. 115 (1988), 127-165.

[8] Sklyanin E., Bispectrality and separation of variables in multiparticle hypergeometric systems, Talk given at the Workshop 'Quantum Integrable Discrete Systems', Cambridge, England, March 23-27, 2009.

[9] Sklyanin E., Bispectrality for the quantum open Toda chain, J. Phys. A: Math. Theor. 46 (2013), 382001, 8 pages, arXiv: 1306.0454 .

[10] Sutherland B., Beautiful models: 70 years of exactly solved quantum many-body problems, World Sci. Publ. Co., Inc., River Edge, NJ, 2004. 